\title{
Anticancer and Apoptotic Effects of Ectoine and Hydroxyectoine on Non-Small Cell Lung Cancer cells: An in-vitro Investigation
}

Mojgan Sheikhpour,2,*, Akram Sadeghi ${ }^{3}$, Fatemeh Yazdian ${ }^{4}$, Abolfazl Movafagh $^{5}$, Ali Mansoori ${ }^{4}$

${ }^{1}$ Department of Mycobacteriology and Pulmonary Research, Pasteur Institute of Iran, Tehran, Iran

${ }^{2}$ Microbiology Research Center, Pasteur Institute of Iran, Tehran, Iran

${ }^{3}$ Department of Microbial Biotechnology, Agricultural Biotechnology Research Institute of Iran (ABRII), Agricultural Research, Education and Extension Organization (AREEO), Karaj, Iran

${ }^{4}$ Department of Life Science Engineering, Faculty of New Sciences and Technologies, University of Tehran, Tehran, Iran

${ }^{5}$ Department of Medical Genetics, Cancer Research Center, Shohada Hospital, School of Medicine, Shahid Beheshti University of Medical Science, Tehran, Iran

*Corresponding author: Mojgan Sheikhpour, Department of Mycobacteriology and Pulmonary Research, Pasteur Institute of Iran, No. 69, Pasteur Ave., Post Code: 1316943551, Tehran, Iran. Tel: +982164112285; Fax: +982164112313;

DOI: 10.30699/acadpub.mci.3.2.14 E-mail:m_sheikhpour@pasteurac.ir

Submitted: 4 February 2019

Revised: 27 February 2019

Accepted: 11 March 2019

e-Published: 1 April 2019

Keywords:

Ectoine

Hydroxyectoine

Lung Cancer

Cell Line

Apoptosis

\section{Abstract}

Introduction: Streptomyces manage the osmotic stress by collecting compatible solutes or osmolytes. Ectoine, 1,4,5,6-tetrahydro-2-methyl-4-pyrimidine carboxylic acid, and 5-hydroxyectoine are among the most common osmolytes of this bacteria and have an active role in protecting the genetic material of this microorganism as well as providing resistance to environmental pressures. In this research, the antiproliferative and apoptotic effects of ectoine and 5hydroxyectoine on human non-small cell lung cancer (NSCLC) cell line (QU-DB) are investigated. Methods: QU-DB cells were treated by five doses of ectoine and hydroxyectoine for 12, 24 and 48 hours, and the cytotoxic properties were evaluated by MTT assay. Then, the DAPI fluorescent staining was carried out for monitoring of the cells' nuclear morphology. Recognition and quantification of the apoptosis were done; using the Annexin-V-Fluos technique.

Results: It has been verified that both ectoine and hydroxyectoine persuaded apoptosis on QUDB cells in a dose-dependently manner. Also, no cytotoxicity effects of these compatible solutes were observed on normal cells (HFLF-PI5).

Conclusions: Ectoine and hydroxyectoine are two natural metabolites which induce apoptosis in lung cancer cells. They don't have any toxic effect on normal cells and can be used as auxiliary drugs or even as a medicine for the treatment of lung cancer after further optimization of the formulations.

(C) 2019. Multidisciplinary Cancer Investigation

\section{INTRODUCTION}

Lung cancer is the foremost wide-reaching cancerrelated deaths that may be caused by cigarette smoking, chemical exposure, radon gas, and air pollution [1-3]. Lung cancer does not usually display 
any important symptoms before the metastasis stage. So, on time diagnosis would be the main issue for increasing the survival of patients with lung cancer [4]. Remedial methods which have been broadly used for the treatment of lung cancer include chemotherapy, radiotherapy, and surgery as well as several drugs such as cisplatin, etoposide, celecoxib, and paclitaxel [5-9]. With all this, there are several disadvantages using these techniques. For example, chemotherapy has definite side effects such as severe toxicity [10]. Recent studies have shown that herbal drugs are attractive manners for medical treatment of lung cancer with few side effects. The killing of the cancerous cell via the programmed cell death pathway; known as apoptosis, is the best goal of anticancer agent's usage with an insignificant side effect on normal cells. Various studies have shown that people with cancer usually have an interest in using plants or herbal products to achieve health and recovery [11]. Moreover, numerous natural compounds and metabolites of plants, fungi, and bacteria are useful and effective in sensitizing the conventional agents [12].

Various microorganisms store a particular class of biological osmolytes, the compatible solutes, to tolerate high osmolarity of ecological niches and prevent cellular hydration and turgor [1315]. Along with their mentioned applications as osmoprotectants, compatible solutes are also protein-stabilizer to maintain the proper folding of polypeptides both in vitro and in vivo under denaturing conditions [16-19]. So, according to the documentation, they are mentioned as chemical chaperones, and as protectants against heat and cold stress [16, 20-23]

The ectoine [(S)-2- methyl-1,4,5,6tetrahydropyrimidine-4-carboxylic acid], tetrahydropyrimidines ectoine, and its hydroxylated derivative 5-hydroxyectoine [(4S,5S)-5- hydroxy-2methyl-1,4,5,6-tetrahydropyrimidine -4-carboxylic acid] are among the most commonly bacterially used compatible solutes [23].

They also are the most frequent osmolytes which are usually found in Streptomyces [24]. Some studies presented promising results about the preventive effect of ectoine on the induction of antiinflammation and anti-apoptotic signaling in the immune system [24].

Although ectoine has been introduced as a defensive agent in animals and humans [25], there are limited documents to prove their therapeutical potentials. As described, in the present research, the goal was to investigate the antiproliferation effects of ectoine and hydroxyectoine in QU-DB cells as an in vitro model of non-small cell lung cancer (NSCLC).

This research was designed to provide future therapeutic strategies for clinical management of lung cancer.

\section{METHODS}

\section{Chemotherapeutic Agent and Cell Culture}

Ectoine and hydroxyectoine were attained from the BIOTREND chemical AG (Zurich, Switzerland). Concentrations of 1 to $20 \mu \mathrm{mol} / \mathrm{L}$ were prepared from each of them. Human lung carcinoma cell line (QU-DB) was prepared from the National Cell Bank of Iran (NCBI) and was cultured in Dulbecco's Modified Eagle's medium (DMEM); complemented with $10 \%$ fetal bovine serum in a moistened atmosphere of $5 \% \mathrm{CO} 2$ and $95 \%$ air at $37^{\circ} \mathrm{C}$. Human lung cell line (HFLF-PI5) was used as the control group (Data not showed). HFLF-PI5 was cultured in RPMI in the same conditions.

\section{Cell Viability}

QU-DB cells were treated in triplicate for each test with different concentrations (1-20 micromolar) of ectoine and hydroxyectoine. To determine the effect of this compatible solutes on cell proliferation, the MTT assay was done after the treatment. Accordingly, $10 \mu \mathrm{l}$ of MTT reagent; 3-(4,5-dimethylthiazol-2yl)-2,5-diphenyl tetrazolium bromide), was added to each well. Following another incubation period of 4 hours at $37^{\circ} \mathrm{C}$, the supernatant was removed from each well. The colored formazan crystals, which were produced from MTT, were dissolved in Dimethyl sulfoxide (DMSO; sigma. USA) and absorbance was then measured at $570 \mathrm{~nm}$ by an ELISA plate reader (ELX800TM, USA).

\section{DAPI Staining}

The blue-fluorescent DAPI was used for the morphological detection of ectoine and hydroxyectoine-induced apoptotic cells according to the standard protocol [12].

\section{Flow Cytometry}

For the recognition and quantification of apoptosis and its distinction from necrosis, the AnnexinV-Fluos staining method was used. Accordingly, 
$20 \mu 1$ of Annexin-V-Fluos labeling reagent was prediluted in $1 \mathrm{ml}$ incubation buffer and added to 20 $\mu l$ of propidium iodide (PI) solution. The cell pellets which were treated by ectoine and hydroxyectoine were resuspended in $100 \mu 1$ of Annexin-V-Fluos solution after incubation for $15 \mathrm{~min}$ at $25^{\circ} \mathrm{C}$. The evaluation was done on a flow cytometer (BD, USA) and data analysis was done by a Flowmax software $[26,27]$.

\section{RESULTS}

\section{MTT Assay}

As shown in Figure 1 and Figure 2, the repressed proliferation of these cancer cell lines were significantly different in concentrations ranging from 1 to $2 \mu \mathrm{mol} / \mathrm{L}$ and during the 24 hours of incubation.

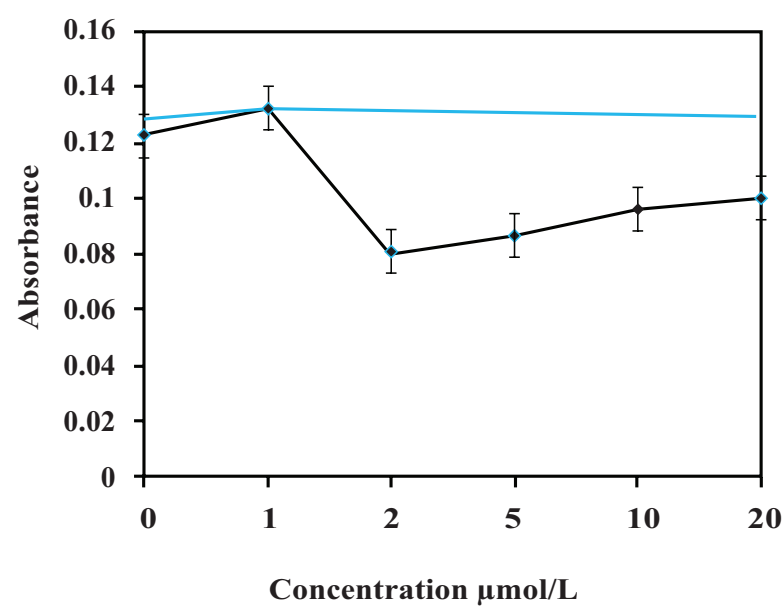

Figure 1: The Absorbance of QU-DB Cells at $570 \mathrm{~nm}$ After 24 Hours of Treatment With Different Concentrations of Ectoine

The blue line indicates the absorbance of normal cells in a similar condition.

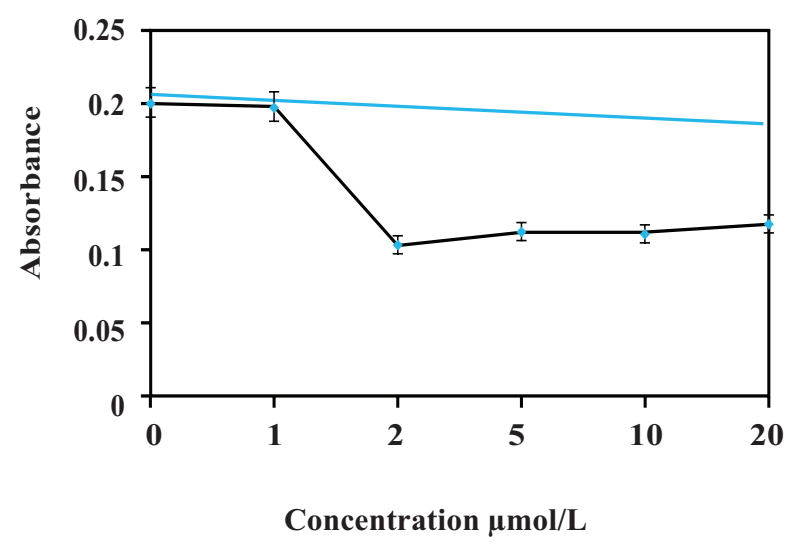

Figure 2: The Absorbance of QU-DB Cells at $570 \mathrm{~nm}$ After 24 Hours of Treatment With Different Concentrations of Hydroxyectoine

The blue line indicates the absorbance of normal cells in a similar condition.
The results showed that the minimum percentage of cell viability after treatment with various concentrations of ectoine was $77 \%$ being obtained at the concentration of $2 \mu \mathrm{mol} / \mathrm{L}$. After treatment with different concentrations of hydroxyectoine, the minimum percentage of cell viability was $67.7 \%$ at the same concentration.

\section{DAPI Staining}

As displayed in Figure 3, the extreme rise in the number of apoptotic cells were found in the same concentration of $2 \mathrm{mM}$ for both ectoine and hydroxyectoine.

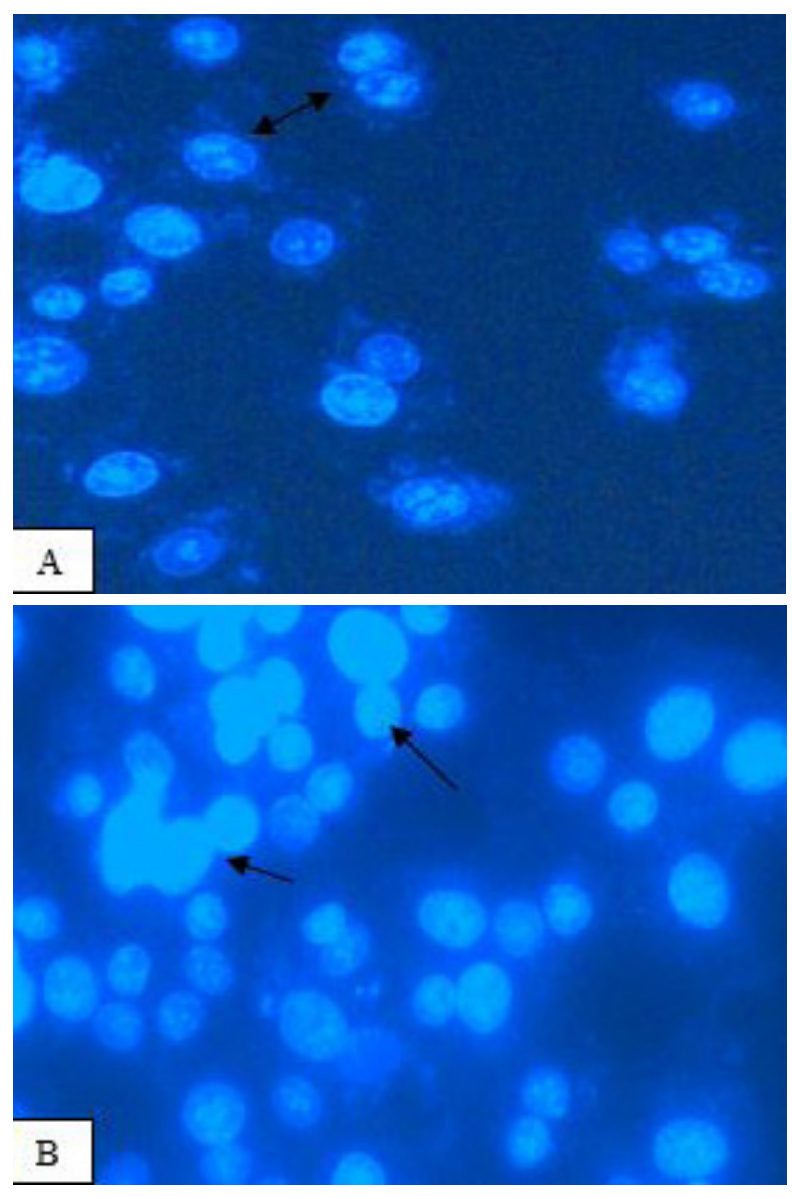

Figure 3: A) DAPI Staining of QU-DB Cells Affected by Ectoine Treatment for 24 Hours; B) DAPI Staining of QU-DB Cells Treated With Hydroxyectoine for 24 Hours

$\rightarrow$ indicates normal cells, $\leftrightarrow$ indicates apoptotic cells

Flow Cytometry Analysis of Apoptotic Cancer Cells TheFlow Cytometric analysis evidentlydiscriminates standard (living) cells with low Annexin and low PI staining, apoptotic cells with high Annexin and low PI staining, and necrotic cells with high Annexin and high PI staining. As represented in Figure 4, the maximum apoptosis induction was observed in the concentration of $2 \mathrm{mM}$ for both ectoine (20.97\%) and hydroxyectoine $(20.97 \%)$. 


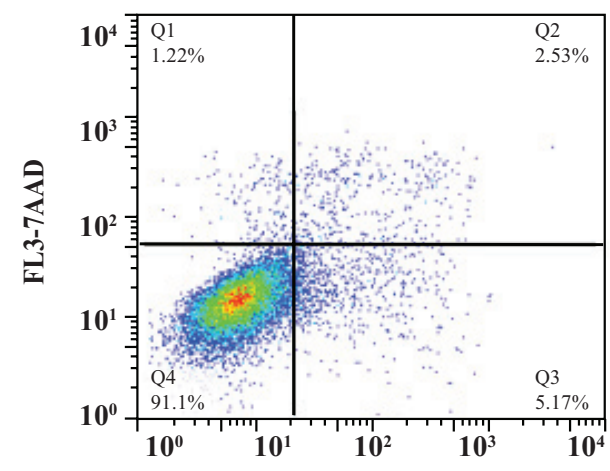

A)

FL2-Annexin-PE

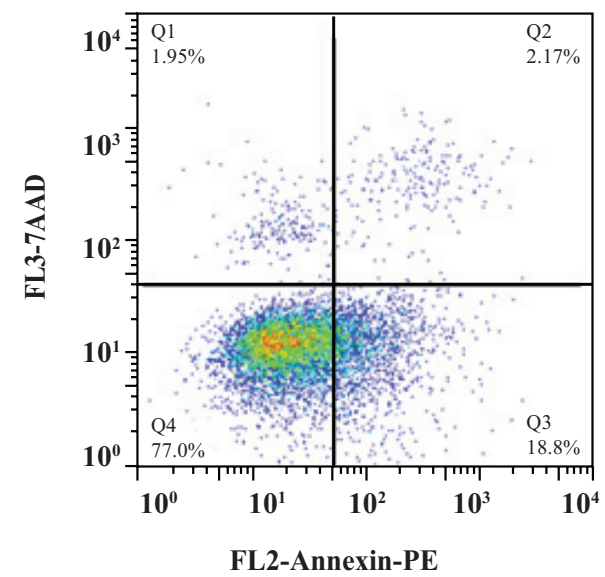

B)

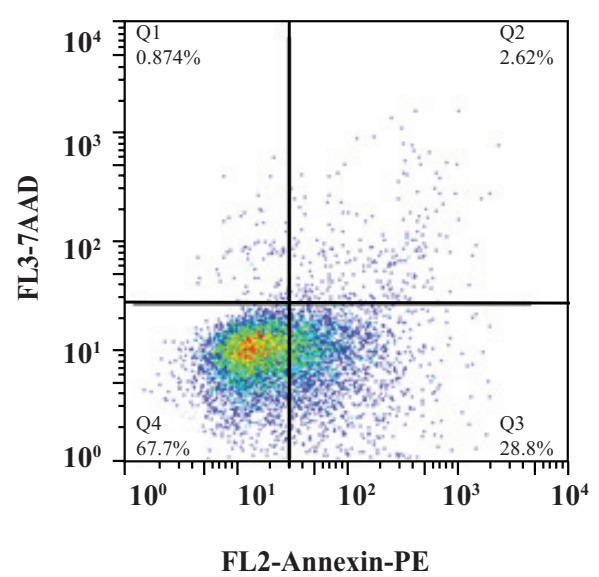

C)

Figure 4: Flow Cytometry Analysis of Apoptotic QU-DB Cells

A) Control; B) Cells Treated by Ectoine; C) Cells Treated by Hydroxyectoine.

\section{DISCUSSION}

The metabolites that are secreted by some microorganisms for their survival and protection, may have beneficial effects for other organisms. For example, Streptomyces produce and accumulate compatible solutes such as ectoine and hydroxyectoine for environmental stresses protection. The widespread expansion of natural metabolites such as ectoines with biologic activity and therapeutic potentialshas increased the attention of scientists. Due to the significant increase in demand for these new therapeutic agents, efforts have been made to optimize the production processes of such metabolites and allow their applications to protect macromolecules, cells, and tissues [28].

Considering the fact that compatible solutes have minor toxic potentials, this group of substances may be used in clinical trials to evaluate their efficacy in the prevention and management of lung cancer as a substitute for old-style methods. Today, according to the new approaches in the field of biomimetics in drug delivery and treatment, these natural metabolites can be used with excellent efficiency, but without noticeable side effects [29, 30]. Also, they can be used in the design and manufacture of new anticancer nano-bio hybrid drugs [31]. It was demonstrated that ectoine and hydroxyectoine, dose-dependently reduced the viability of QU-DB cell line, the human lung cancer cell. Briefly, both ectoine and hydroxyectoine have cytotoxic effects on these cancer cells at $2 \mathrm{mM}$ of concentration. In addition, hydroxyectoine has more cytotoxic effects on QU-DB cells than ectoine.

Previous studies have shown that ectoine activates the MAPK A ERK1/2, intracellular signaling pathways, and cell-cell interaction [32]. Although ectoine is used as an anti-inflammatory agent in pharmaceutical sprays, the apoptotic study 
on lung cancer cells was conducted for the first time. Biological, genetical, and in vivo studies will allow a deeper understanding of ectoine and hydroxyectoine roles in cell signaling pathways. Bioprocess and metabolic engineering strategies are needed prior to the clinical applications of ectoines. So, further studies such as cell signaling and in vivo investigations are under process; using controlled systems like bioreactors for process optimization.

Based on the new findings of this in vitro research, we demonstrated for the first time that both ectoine and hydroxyectoine inhibited the rapid and irregular growth of lung cancer cells. However, further studies are required in animal models to find the genetic pathways involved in apoptosis.

\section{ACKNOWLEDGMENTS}

The authors acknowledge the technical and equipmental supports of $\mathrm{dr}$ seyed davar siadat and other colleagues in department of Mycobacteriology and Pulmonary Research, Pasteur Institute of Iran.

\section{CONFLICT OF INTEREST}

The authors declare that they have no conflict of interest.

\section{ETHICS APPROVAL}

Not applicable.

\section{REFERENCES}

1. Gorlova OY, Weng SF, Zhang Y, Amos CI, Spitz MR. Aggregation of cancer among relatives of never-smoking lung cancer patients. Int J Cancer. 2007;121(1):111-8. DOI: 10.1002/ijc.22615 PMID: 17304511.

2. Catelinois O, Rogel A, Laurier D, Billon S, Hemon D, Verger $\mathrm{P}$, et al. Lung cancer attributable to indoor radon exposure in france: impact of the risk models and uncertainty analysis. Environ Health Perspect. 2006;114(9):1361-6. DOI: 10.1289/ehp.9070 PMID: 16966089.

3. Kabir Z, Bennett K, Clancy L. Lung cancer and urban air-pollution in Dublin: a temporal association? Irish Med J. 2007;100(2):367-9.

4. Jeong SJ, Koh W, Kim B, Kim SH. Are there new therapeutic options for treating lung cancer based on herbal medicines and their metabolites? J Ethnopharmacol. 2011;138(3):652-61. DOI: 10.1016/j.jep.2011.10.018 PMID: 22032843.

5. Murray N, Turrisi AT, 3rd. A review of first-line treatment for small-cell lung cancer. J Thorac Oncol. 2006;1(3):2708. PMID: 17409868 .

6. Aruajo AM, Mendez JC, Coelho AL, Sousa B, Barata F, Figueiredo A, et al. Phase II study of celecoxib with cisplatin plus etoposide in extensive-stage small cell lung cancer. Cancer Invest. 2009;27(4):391-6. DOI:

\subsection{0/07357900802232756 PMID: 19266367.}

7. Azim HA, Jr., Ganti AK. Treatment options for relapsed small-cell lung cancer. Anticancer Drugs. 2007;18(3):25561. DOI: 10.1097/CAD.0b013e328011a547 PMID: 17264756.

8. Qin A, Kalemkerian GP. Treatment Options for Relapsed Small-Cell Lung Cancer: What Progress Have We Made? J Oncol Pract. 2018;14(6):369-70. DOI: 10.1200/ JOP.18.00278 PMID: 29894661.

9. Clegg A, Scott DA, Hewitson P, Sidhu M, Waugh N. Clinical and cost effectiveness of paclitaxel, docetaxel, gemcitabine, and vinorelbine in non-small cell lung cancer: a systematic review. Thorax. 2002;57(1):20-8. DOI: 10.1136/ thorax.57.1.20 PMID: 11809985.

10. Broker LE, Giaccone G. The role of new agents in the treatment of non-small cell lung cancer. Eur J Cancer. 2002;38(18):2347-61. PMID: 12460778.

11. Safarzadeh E, Shotorbani SS, Baradaran B. Herbal medicine as inducers of apoptosis in cancer treatment. Adv Pharm Bull. 2014;4(Suppl 1):421. DOI: 10.5681/ apb.2014.062 PMID: 25364657.

12. Sheikhpour M, Ahangari G, Sadeghizadeh M, Deezagi A. A novel report of apoptosis in human lung carcinoma cells using selective agonist of D2-like dopamine receptors: a new approach for the treatment of human nonsmall cell lung cancer. Int J Immunopathol Pharmacol. 2013;26(2):393-402. DOI: 10.1177/039463201302600212 PMID: 23755754.

13. Kempf B, Bremer E. Uptake and synthesis of compatible solutes as microbial stress responses to high-osmolality environments. Arch Microbiol. 1998;170(5):319-30. PMID: 9818351.

14. Storz G, Hengge R. Bacterial stress responses. USA: American Society for Microbiology Press; 2010. DOI: $10.1128 / 9781555816841$

15. Wood JM, Bremer E, Csonka LN, Kraemer R, Poolman B, van der Heide $\mathrm{T}$, et al. Osmosensing and osmoregulatory compatible solute accumulation by bacteria. Comp Biochem Physiol A Mol Integr Physiol. 2001;130(3):437-60. DOI: 10.1016/S1095-6433(01)00442-1.

16. Bayles DO, Wilkinson BJ. Osmoprotectants and cryoprotectants for Listeria monocytogenes. Lett Appl Microbiol. 2000;30(1):23-7. PMID: 10728555.

17. Bourot S, Sire O, Trautwetter A, Touze T, Wu LF, Blanco C, et al. Glycine betaine-assisted protein folding in a lysA mutant of Escherichia coli. J Biol Chem. 2000;275(2):1050-6. DOI: $10.1074 / \mathrm{jbc} .275 .2 .1050$ PMID: 10625645.

18. Ignatova $Z$, Gierasch LM. Inhibition of protein aggregation in vitro and in vivo by a natural osmoprotectant. Proc Natl Acad Sci U S A. 2006;103(36):13357-61. DOI: 10.1073/ pnas.0603772103 PMID: 16899544.

19. Street TO, Bolen DW, Rose GD. A molecular mechanism for osmolyte-induced protein stability. Proc Natl Acad Sci U S A. 2006;103(38):13997-4002. DOI: 10.1073/ pnas.0606236103 PMID: 16968772.

20. Chattopadhyay MK, Kern R, Mistou MY, Dandekar AM, Uratsu SL, Richarme G. The chemical chaperone proline relieves the thermosensitivity of a dnaK deletion mutant 
at 42 degrees C. J Bacteriol. 2004;186(23):8149-52. DOI: 10.1128/JB.186.23.8149-8152.2004 PMID: 15547289.

21. Diamant S, Eliahu N, Rosenthal D, Goloubinoff P. Chemical chaperones regulate molecular chaperones in vitro and in cells under combined salt and heat stresses. J Biol Chem. 2001;276(43):39586-91. DOI: 10.1074/jbc.M103081200 PMID: 11517217.

22. Holtmann G, Bremer E. Thermoprotection of Bacillus subtilis by exogenously provided glycine betaine and structurally related compatible solutes: involvement of Opu transporters. J Bacteriol. 2004;186(6):1683-93. DOI: 10.1128/ jb.186.6.1683-1693.2004 PMID: 14996799.

23. Hoffmann T, Bremer E. Protection of Bacillus subtilis against cold stress via compatible-solute acquisition. J Bacteriol. 2011;193(7):1552-62. DOI: 10.1128/JB.0131910 PMID: 21296969.

24. Sydlik U, Peuschel H, Paunel-Gorgulu A, Keymel S, Kramer U, Weissenberg A, et al. Recovery of neutrophil apoptosis by ectoine: a new strategy against lung inflammation. Eur Respir J. 2013;41(2):433-42. DOI: $\underline{10.1183 / 09031936.00132211}$ PMID: 23100492.

25. Bownik A, Stepniewska Z. Ectoine as a promising protective agent in humans and animals. Arh Hig Rada Toksikol. 2016;67(4):260-5. DOI: 10.1515/aiht-2016-67-2837 PMID: 28033102 .

26. Vermes I, Haanen C, Steffens-Nakken H, Reutellingsperger C. A novel assay for apoptosis flow cytometric detection of phosphatidylserine expression on early apoptotic cells using fluorescein labelled annexin V. J Immunol Methods. 1995; 184(1):39-51.

27. Fadok VA, Savill JS, Haslett C, Bratton DL, Doherty DE, Campbell PA, et al. Different populations of macrophages use either the vitronectin receptor or the phosphatidylserine receptor to recognize and remove apoptotic cells. J Immunol. 1992;149(12):4029-35. PMID: 1281199.

28. Pastor JM, Salvador M, Argandona M, Bernal V, Reina-Bueno M, Csonka LN, et al. Ectoines in cell stress protection: uses and biotechnological production. Biotechnol Adv. 2010;28(6):782-801. DOI: 10.1016/j.biotechadv.2010.06.005 PMID: 20600783.

29. Baker TK. Methods and reagents for maintaining the viability of cancer cells in surgically removed tissue. Google Patents; 2015.

30. Sheikhpour M, Barani L, Kasaeian A. Biomimetics in drug delivery systems: A critical review. J Control Release. 2017;253:97-109. DOI: 10.1016/j.jconrel.2017.03.026 PMID: 28322976.

31. Sheikhpour M, Golbabaie A, Kasaeian A. Carbon nanotubes: A review of novel strategies for cancer diagnosis and treatment. Mater Sci Eng C Mater Biol Appl. 2017;76:1289304. DOI: 10.1016/j.msec.2017.02.132 PMID: 28482496.

32. Sydlik U, Gallitz I, Albrecht C, Abel J, Krutmann J, Unfried K. The compatible solute ectoine protects against nanoparticle-induced neutrophilic lung inflammation. Am J Respir Crit Care Med. 2009;180(1):29-35. DOI: 10.1164/ rccm.200812-19110C PMID: 19324973. 